\title{
Macrophages promote progression of lung cancer by regulating ubiquitin-specific peptidase 17 in cancer cells
}

\author{
Chih-Hao Lu, Chao-Yang Lai and Tsung-Hsien Chuang*
}

Immunology Research Center, National Health Research Institutes, Taiwan

Tumor-associated macrophages (TAMs) are stromal cells in the tumor microenvironment that affect the progression of tumor development. Macrophages can be divided into different subsets based on their functions. A simplistic model is that M1 (or classically activated) macrophages favor the immunosurveillance of malignant cells and M2 (or alternatively activated) macrophages exert immunosuppressive effects and perform protumoral functions. TAMs are not a single uniform population. They exhibit features that are intermediate between the M1 and M2 macrophage phenotypes. In some cancer types such as lung cancer, extensive accumulation of TAMs is often associated with poor prognosis. In addition to the regulation of immunological responses, TAMs promote tumor progression through the regulation of cancer cell proliferation, invasion, and metastasis. Therefore, the molecular mechanisms underlying the interaction between macrophages and cancer cells to build a protumoral microenvironment were investigated with the goal of developing antitumor strategies [1-4].

Ubiquitination is a post-translational protein modification process that is involved in several cellular functions. Specific ubiquitination of a target protein is mediated by E3 ubiquitin-protein ligase and is counteracted by deubiquitinases. Dysfunction of this ubiquitinationdeubiquitination process has been linked to human diseases, including cancers [5-7]. Ubiquitin-specific protease 17 (UPS17, also termed as DUB3) is a cytokine-inducible deubiquitinase [8]. A recent study demonstrated a high expression of USP17 in lung cancers. The expression of this deubiquitinase was found to increase in parallel with the increase in cancer stages, suggesting that USP17 expression is associated with poor prognosis in lung cancers [9]. Consistent with these findings, the analysis of the Oncomine database revealed that high levels of USP17 expression were also observed in cervical, gastric, and breast cancers (Figure 1). Moreover, results from the cBioPortal database analysis demonstrated a correlation between high USP17 expression and a low survival rate in patients with thyroid carcinoma (Figure 2).

Different molecular mechanisms underlying the protumoral function of USP17 have been reported. Earlier studies have suggested
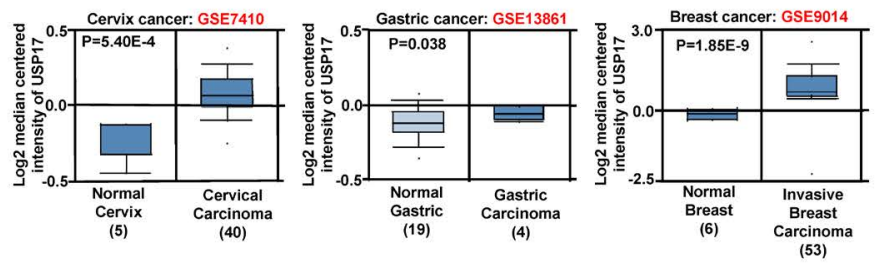

Figure 1. High ubiquitin-specific peptidase 17 (USP17) expression in different cancers Data of USP17 expression levels in the normal and cancer tissues as indicated were acquired from data sets in the Oncomine database for analysis. Red: accession number for the data set. Tissue sample numbers are shown in parentheses.

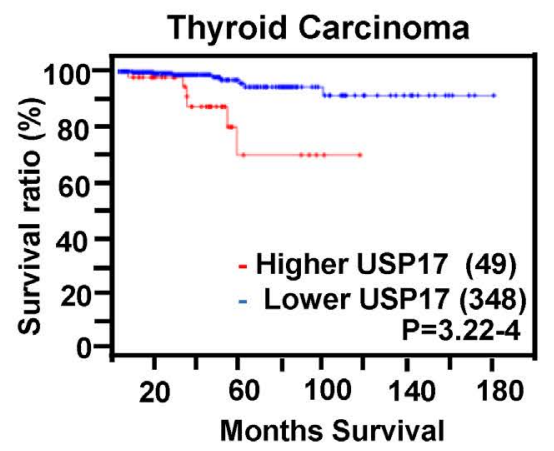

Figure 2. Correlation between high ubiquitin-specific peptidase 17 expression and poor prognosis in thyroid carinoma

Kaplan-Meier plot of the survival rate of patients with thyroid carcinoma stratified by low and high USP17 expression levels. Data were acquired for analysis from the cBioPortal data set: TCGA. Expression levels greater than 1SD of mean were defined as higher USP17 and others were lower USP17 expression. Tissue sample numbers are shown in parentheses.

that this deubiquitinase promotes oncogenic transformation through the regulation of cell cycle progression. USP17 expression is required for G1-S progression through the regulation of the cyclin-dependent kinase inhibitors $\mathrm{p} 21$ and $\mathrm{p} 27$ [10]. The deubiquitinase activity of USP 17 protects Cdc25A from proteasomal degradation and promotes the celltransforming ability [11]. More recent studies have demonstrated that USP17 is involved in the regulation of the epithelial-mesenchymal transition (EMT) phenotype and cancer invasion by stabilizing snaill, slug, twist, and SMAD4 through its deubiquitination activity [12-15]. Another study reported that USP17 deubiquitinates a bromodomain and an extraterminal domain (BET) protein, BRD4, to promote tumor progression [16].

The tumor microenvironment contains abundant cytokines, and TAMs are a major source of these cytokines. Therefore, the expression and function of USP17 in cancer cells in mediating the protumoral effect of macrophages were investigated [9]. The expression of USP17 was elevated in correlation with the expression of inflammatory markers and macrophage markers in lung cancers. Both the M1 type and the M2 type of macrophages promoted the expression of USP17 in lung cancer cells, and this effect was caused due to the cytokines produced

*Correspondence to: Tsung-Hsien Chuang, Immunology Research Center, National Health Research Institutes, 35 Keyan Road, Zhunan, Miaoli County 35053, Taiwan, E-mail: thchuang@nhri.org.tw

Key words: macrophage, inflammation, stemness, ubiquitin-specific peptidase 17 Received: September 28, 2018; Accepted: October 12, 2018; Published: October 16, 2018 
by these macrophages. An increased expression of USP17 enhances inflammation and stemness in cancer cells by stabilizing key signaling molecules and transcription factors, including NF- $\kappa \mathrm{B}$-inducing kinase (NIK), c-Rel, and interferon regulatory factor 5 (IRF5). Proteolytic degradation of these proteins is promoted by a TNF-receptorassociated factor 2 (TRAF2)/TRAF3 complex. USP17 contains TRAF2and TRAF3-binding domains, which allow it to interact and disrupt the protein degradation capability of this TRAF2/TRAF3 complex. In addition, the expression of USP17 in cancer cells promotes macrophage recruitment and cytokine production by macrophages. Animal studies have demonstrated that there was a reduction in the tumor growth rate, USP17 expression, and inflammatory cytokine production in tumors grown in macrophage-depleted mice compared with that in control mice. Moreover, there was a faster tumor growth rate, increased leukocyte infiltration, and inflammation- and stemness-associated gene expressions in tumors derived from USP17-overexpressed lung cancer cells compared with those derived from control cancer cells [9]. These results suggested a positive role of USP17 expression in lung cancer cells in the protumoral function of macrophages.

\section{References}

1. Quatromoni JG, Eruslanov E (2014) Tumor-associated macrophages: function, phenotype, and link to prognosis in human lung cancer. Am J Transl Res 4:376-389. [Crossref]

2. Noy R, Pollard JW (2014) Tumor-associated macrophages: from mechanisms to therapy. Immunity 41: 49-61. [Crossref]

3. Conway EM, Pikor LA, Kung SH, Hamilton MJ, Lam S, et al. (2016) Macrophages, Inflammation, and Lung Cancer. Am J Respir Crit Care Med 193: 116-130. [Crossref]

4. Shapouri-Moghaddam A, Mohammadian S, Vazini H, Taghadosi M, Esmaeili SA, et al. (2017) Macrophage plasticity, polarization, and function in health and disease. $J$ Cell Physiol 233: 6425-6440. [Crossref]
5. Kessler BM (2013) Ubiquitin - omics reveals novel networks and associations with human disease. Curr Opin Chem Biol 17: 59-65. [Crossref]

6. Gallo LH, Ko J, Donoghue DJ (2017) The importance of regulatory ubiquitination in cancer and metastasis. Cell Cycle 16: 634-648. [Crossref]

7. Inoue $Y$, Itoh $Y$, Sato K, Kawasaki F, Sumita C, et al. (2016) Regulation of EpithelialMesenchymal Transition by E3 Ubiquitin Ligases and Deubiquitinase in Cancer. Curr Cancer Drug Targets 16: 110-118. [Crossref]

8. Lim KH, Ramakrishna S, Baek KH (2013) Molecular mechanisms and functions of cytokine-inducible deubiquitinating enzymes. Cytokine Growth Factor Rev 24:427431. [Crossref]

9. Lu CH, Yeh DW, Lai CY, Liu YL, Huang LR, et al. (2018) USP17 mediates macrophage-promoted inflammation and stemness in lung cancer cells by regulating TRAF2/TRAF3 complex formation. Oncogene.

10. McFarlane C, Kelvin AA, de la Vega M, Govender U, Scott CJ, et al. (2010) The deubiquitinating enzyme USP17 is highly expressed in tumor biopsies, is cell cycle regulated, and is required for G1-S progression. Cancer Res 70: 3329-3339. [Crossref]

11. Pereg Y, Liu BY, O'Rourke KM, Sagolla M, Dey A, et al. (2010) Ubiquitin hydrolase Dub3 promotes oncogenic transformation by stabilizing Cdc25A. Nat Cell Biol 12: 400-406. [Crossref]

12. Liu T, Yu J, Deng M, Yin Y, Zhang H, et al. (2017) CDK4/6-dependent activation of DUB3 regulates cancer metastasis through SNAIL1. Nat Commun 8: 13923. [Crossref]

13. Wu Y, Wang Y, Lin Y, Liu Y, Wang Y, et al. (2017) Dub3 inhibition suppresses breast cancer invasion and metastasis by promoting Snaill degradation. Nat Commun 8: 14228. [Crossref]

14. Lin Y, Wang Y, Shi Q, Yu Q, Liu C, et al. (2017) Stabilization of the transcription factors slug and twist by the deubiquitinase dub3 is a key requirement for tumor metastasis. Oncotarget 8: 75127-75140. [Crossref]

15. Song C, Liu W, Li J (2017) USP17 is upregulated in osteosarcoma and promotes cell proliferation, metastasis, and epithelial-mesenchymal transition through stabilizing SMAD4. Tumour Biol. [Crossref]

16. Jin X, Yan Y, Wang D, Ding D, Ma T, et al. (2018) DUB3 Promotes BET Inhibitor Resistance and Cancer Progression by Deubiquitinating BRD4. Mol Cell 71: 592-605. [Crossref]

Copyright: (C2018 Chih-Hao L. This is an open-access article distributed under the terms of the Creative Commons Attribution License, which permits unrestricted use, distribution, and reproduction in any medium, provided the original author and source are credited. 